MKG-Chirurg 2015 $\cdot 8: 73$

DOI 10.1007/s12285-015-0010-9

Online publiziert: 28. April 2015

(c) Springer-Verlag Berlin Heidelberg 2015

\author{
H.-F. Zeilhofer \\ Klinik für Mund-, Kiefer- und Gesichtschirurgie, Universitätsspital Basel, Basel
}

\title{
Klinische Ethik
}

\section{Hilfe zur Entscheidungsfindung}

Liebe Kolleginnen und Kollegen,

in den Leitthemen unserer Zeitschrift Der $M K G$-Chirurg versuchen wir, wichtige Bereiche unseres Fachgebiets auf dem neuesten Stand der Technik aus unterschiedlichen Perspektiven darzustellen. Bei dem rapiden Zuwachs an wissenschaftlichen Erkenntnissen ist es nach Abschluss eines Themenzyklus bereits höchste Zeit, wieder von vorn zu beginnen und mit alten Irrtümern aufzuräumen.

Darunter Ethik als Leitthema zu behandeln, ist zwar ein Novum, aber sicher kein Luxus. Wir haben als Ärzte im Laufe unserer Berufsjahre einen Sinn für die Unterscheidung von Gutem und Schlechtem entwickelt. Doch wie weit trägt uns dieses subjektiv-moralische Empfinden, und wann und wo stoßen wir damit an Grenzen?

\section{1) Bei Entscheidungen im beruflichen Alltag und in der Forschung das Richtige tun}

Die Medizinethik ist eine junge Disziplin im Kanon der wissenschaftlichen Disziplinen; sie will uns nicht mit moralisch erhobenem Zeigefinger belehren, sondern das geistige Rüstzeug bieten, damit wir (nicht nur) bei schwierigen Entscheidungen während unseres beruflichen Alltags das Richtige tun. So helfen uns z. B. die Fragen eines hinzugezogenen Ethikbeirats bei der Entscheidung, ob und wann man eine Therapie abbrechen soll und darf.

Gerade weil die Gesundheit von der Gesellschaft als eines der wertvollsten Güter allgemein akzeptiert wird, ist sie in ihrem Verhältnis zu anderen Gütern wie Wohlstand oder Bildung und Glück immer wieder neu zu definieren und abzu- grenzen. Aus diesem Grund werden wir in den nächsten Heften für unseren Beruf wichtige ethische Themen als Einzelbeiträge einfügen.

Wir beginnen mit einem Beitrag zur Radiologie im Fachgebiet. Wir haben die Berechtigung, eigenständig Röntgengeräte zu betreiben und über die Art der bildgebenden Untersuchung, die wir für unsere Patienten einsetzen, selbst zu entscheiden. Der Gesetzgeber hat die Vorschriften für die Fortbildung und den Strahlenschutz geregelt, was der Qualitätssicherung zweifelsfrei dient. Die Aspekte der Fortbildung von allen MKG-Chirurgen und Zahnärzten, die selbst keine Geräte betreiben, zu erörtern sowie das komplexe Thema unter den unterschiedlichen Gesichtspunkten wie Ökonomie, technischem Fortschritt und Patientensicherheit, Interessenkonflikten zwischen Versicherern, Leistungserbringern und Patienten gedanklich anzustoßen, ist uns ein Anliegen.

Auch im Bereich von Wissenschaft und Forschung wurden in den letzten Jahren ethische Empfehlungen erarbeitet, die das Verhältnis zur Industrie oder das Publizieren der wissenschaftlichen Ergebnisse behandeln.

Ein wichtiger ethischer Aspekt ist die transparente Publikation aller positiven und negativen Forschungsergebnisse. Nur durch die Veröffentlichung aller Ergebnisse können aus den Ergebnissen der optimale Nutzen für die Patienten gewonnen und die unnötige Wiederholung gleicher oder ähnlicher Forschungsvorhaben verhindert werden.

In der Forschung hat bewusstes wissenschaftliches Fehlverhalten - das nach einschlägigen Untersuchungen allerdings nur etwa $5 \%$ der Arbeiten betrifft - selbstverständlich Konsequenzen, nicht nur für die beteiligten Wissenschaftler, sondern auch für die Gesellschaft. Es führt zu Wissensrückschlägen in der Forschung, zur Verschwendung von Ressourcen, Geld und Zeit, zu unnötigen Tierversuchen und zu Auswirkungen auf die Gesundheit von Patienten. Häufig werden Interessenkonflikte (Auftraggeber einer Studie, persönliches Renommee des Forschers, Fragen der Finanzierung) als Grund für wissenschaftliches Fehlverhalten genannt.

Es kann vorkommen, dass Studien nicht die gewünschten Ergebnisse zeigen. Die Veröffentlichung negativer Ergebnisse ist oft nicht einfach, da für Zeitschriften, die eine lange Warteliste für Publikationen haben, positive Studienergebnisse interessanter sind und vorgezogen werden.

Präventiv sollten, wie die Autorinnen B.S. Elger und S. Engel-Glatter betonen, alle Studierenden Pflichtkurse zu Grundsätzen von ",richtigem" und „falschem" Verhalten besuchen und die Universitäten detaillierte Richtlinien zum wissenschaftlichen Fehlverhalten erarbeiten. Auch die Fachgesellschaften haben hier eine Verantwortung, der wir in dieser und in den folgenden Ausgaben Rechnung tragen werden.

Ihr

Wansitorn billeter

Prof. Dr. Dr. Dr. H.-F. Zeilhofer

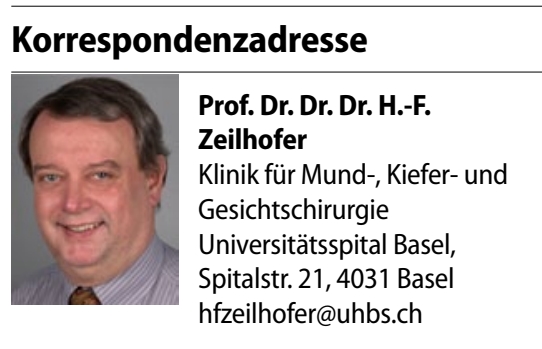

